\title{
Intraoperative Electron Radiotherapy (IOERT) in the Treatment of Primary Breast Cancer
}

\author{
Julia Kaiser $^{\mathrm{a}}$ Roland Reitsamer ${ }^{\mathrm{b}}$ Peter Kopp ${ }^{\mathrm{a}} \quad$ Christoph Gaisberger $^{\mathrm{a}} \quad$ Michael Kopp $^{\mathrm{a}}$ \\ Thorsten Fischer $^{b} \quad$ Franz Zehentmayr $^{\mathrm{a}}$ Felix Sedlmayer ${ }^{\mathrm{a}}$ Gerd Fastner $^{\mathrm{a}}$ \\ ${ }^{a}$ Department of Radiotherapy and Radio-Oncology, Paracelsus Medical University, University Hospital Salzburg, Landeskrankenhaus, \\ Salzburg, Austria; \\ ${ }^{\text {b}}$ Department of Gynecology, Paracelsus Medical University, University Hospital Salzburg, Landeskrankenhaus, Salzburg, Austria
}

\section{Keywords}

Intraoperative electron radiotherapy, IOERT · Electrons · Boost · Breast cancer · Tumor bed

\section{Summary}

IOERT (intraoperative electron radiotherapy) in breast cancer is used either as a boost (10-12 Gy) followed by whole breast irradiation (WBI) or as full-dose partial breast irradiation (PBI, 20-24 Gy) during breast-conserving surgery. IOERT has the longest evidence of all IORT techniques. When administered as a boost, excellent low local recurrence rates were observed in long-term follow-up $>5$ years. Even in high-risk groups like triple-negative or locally advanced breast cancers, IOERT contributes to long-term local control rates of more than $90 \%$. For selected low-risk groups, IOERT as PBI with $21 \mathrm{~Gy}$ seems to be a viable treatment alternative to standard WBI. IOERT has been shown to be advantageous for several reasons: Geographic misses are avoided due to direct visualization of the tumor bed; thus, a high single dose is delivered with utmost precision to small volumes, completely sparing the skin and ensuring good long-term cosmetic outcome. Furthermore, high single doses seem to induce biological mechanisms with verifiable antitumor capability in in-vitro cell-line studies. In addition, IOERT markedly shortens the overall treatment time both in combination with (now mostly hypofractionated) WBI or as a PBI in selected low-risk constellations.

(c) 2018 S. Karger GmbH, Freiburg

\section{Introduction}

In the last decades, local recurrence rates after primary breast cancer treatment have gradually declined, which is attributable to a variety of factors like the increased use of screening mammography, higher quality pathological work-up, improvements in surgical strategies, widespread use of modern systemic therapies, and, last but not least, progress in radiotherapy. For locoregional treatment, breast-conserving surgery (BCS) followed by ipsilateral whole breast irradiation (WBI) is still regarded as the standard of care. However, in recent years, selected cases with a low risk of recurrence have also been considered for partial breast irradiation (PBI) only [1].

Postoperative radiotherapy significantly reduces local recurrence rates - the more pronounced, the more substantially it translates into improved survival. 4 prevented local recurrences after 10 years result in 1 avoided breast cancer death after 15 years $[2,3]$.

\section{Rationale for a Boost and Biology of High Single Doses}

Due to the tumor cell density being highest at a distance of up to $4 \mathrm{~cm}$ from the macroscopic tumor edge $[4,5]$, the highest risk of in-breast recurrences is anticipated in the tumor bed. Thus, following WBI, an additional boost dose to this area significantly reduces local recurrence rates [6]. The most frequently reported boost techniques comprise external electrons or photons with fractionated doses of 10-16 Gy, or interstitial implants as high dose rate brachytherapy.

In contrast to postoperative irradiation, intraoperative radiotherapy (IORT) offers the possibility to apply a high single dose at the time of BCS with utmost precision due to direct visualization.

\section{KARGER}

(c) 2018 S. Karger GmbH, Freiburg

Fax +497614520714
PD Dr. Gerd Fastner

Department of Radiotherapy and Radio-Oncology, Paracelsus Medical University University Hospital Salzburg, Landeskrankenhaus

Müllner Hauptstraße 48, 5020 Salzburg, Austria g.fastner@salk.at 
Fig. 1. Maximum dose (Dmax, 100\%), D90, D45, and their corresponding tissue depths (d) should be specified along the central beam and the clinical axis (in $\mathrm{mm}$ ), respectively.
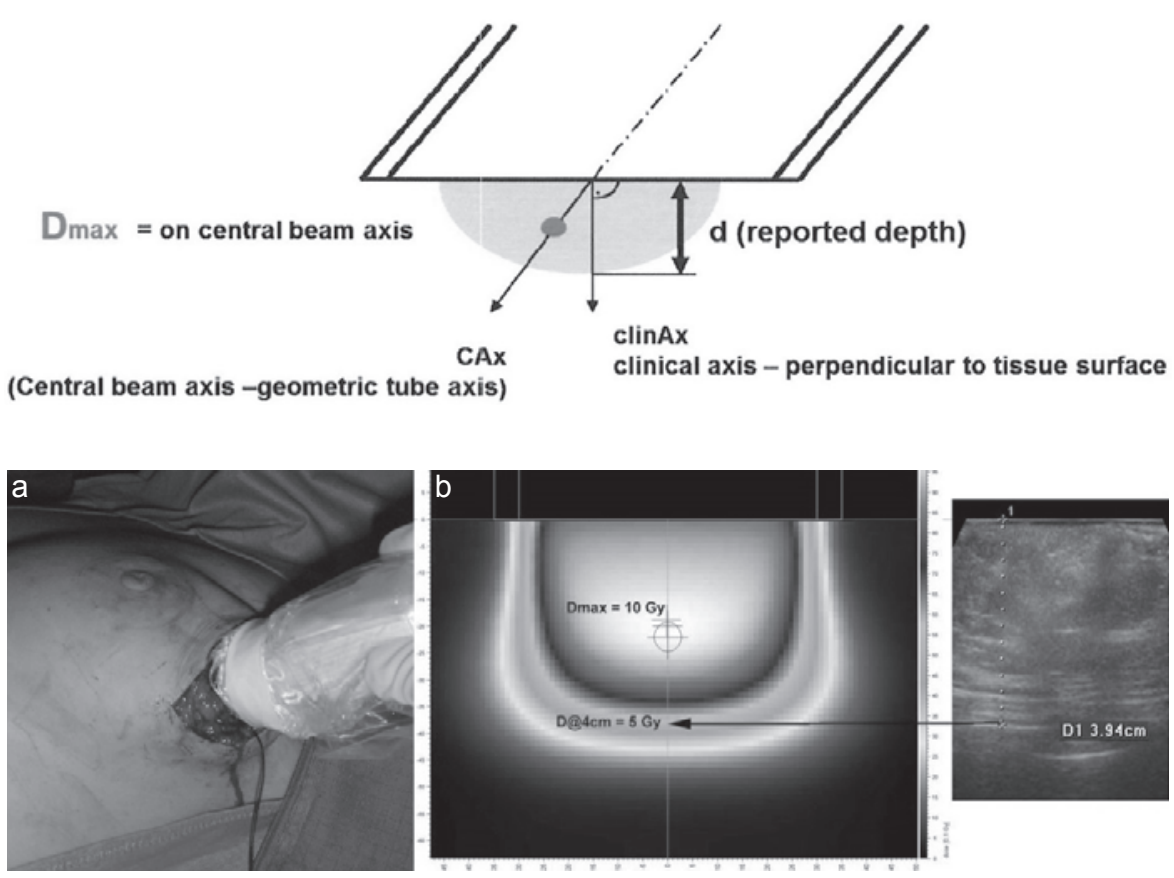

Fig. 2. a Tissue depth calculation by ultrasound and $\mathbf{b}$ corresponding dose profile.

Intraoperative electron radiotherapy (IOERT) was introduced over 20 years ago in 2 small series published by Merrick et al. [7] and DuBois et al. [8].

From a biological point of view, breast cancer cells seem to show a higher sensitivity towards higher doses compared to squamous cell carcinoma (SCC). In order to estimate the effects of different single and total doses in terms of dose response, the linearquadratic model allocates so-called alpha/beta values to various tissue and tumor types. For breast cancer cell lines, Fowler [9] postulated an alpha/beta value of 4 instead of 10 for SCC as far back as 1989. This assumption was strongly supported by the results of the Canadian and British Hypofractionation Trials [10, 11]. Since a lower ratio results in higher sensitivity towards high doses, the use of IORT should be advantageous. For instance, when using an alpha/beta value of 4 , an IORT dose of 10 Gy amounts to a biologically effective dose of 35 , predicting isoefficacy to 24 Gy when applied in single fractions of 2 Gy. However, this model was only tested for single doses below 15 Gy [12]; thus, the prediction of isoeffects of higher doses leaves many questions unanswered and has to be further evaluated. Another supposed 'biological' advantage of IORT is an immediate effect of irradiation on the tumor microenvironment by abrogation of the proliferative cascade induced by wound healing after surgery. In vitro, wound fluid has been described to stimulate tumor cell proliferation and invasion, which can be blocked by high-dose IORT [13, 14]. A further aspect is the prevention of possible residual tumor cell repopulation between surgery and adjuvant radiotherapy ('temporal miss'). Finally, a good oxygenation status of the tumor bed during surgery could also be a factor for enhanced biological effectiveness, which has not yet been investigated [15]. All these cellular and transcellular reactions of irradiated tissues and their particular impact on clonogenic cell inactivation are the subject of ongoing research [16].

\section{IOERT Treatment Concepts}

There are 2 different treatment options for IOERT:

1) IOERT as an anticipated boost followed by WBI;

2) IOERT as full-dose PBI.

\section{Dose Recommendation}

Usual doses for boost IOERT (followed by WBI) are within the range of 9-10 Gy defined as D90 (90\% isodose) $[17,18]$ and have been clinically investigated in all risk constellations $[17,19,20]$ (fig. 1).

In contrast, according to international guidelines [21, 22], PBI strategies including IOERT are recommended for selected lowrisk cases only. In the ELIOT trial as the most recognized study [23], single doses of 21 Gy (D90) were applied as 'full-dose' radiotherapy.

\section{IOERT Procedures}

Intraoperative electrons are applied with mobile or standard linear accelerators. After tumor excision, the surgeon mobilizes part of the remaining breast around the tumor bed to expose the adjacent walls (which form the target volume) to the radiation beam. The appropriate electron energy is determined by the tissue thickness which can be measured using a needle probe, intraoperative ultrasound, or computed tomography. If performed as fulldose PBI, the rib surface is usually protected by lead shielding to keep the exit doses to the bony structures below 5-7 Gy. Dependent on tumor size, surgical clearance, and ductal carcinoma in situ components, electron energy levels commonly range between 4 

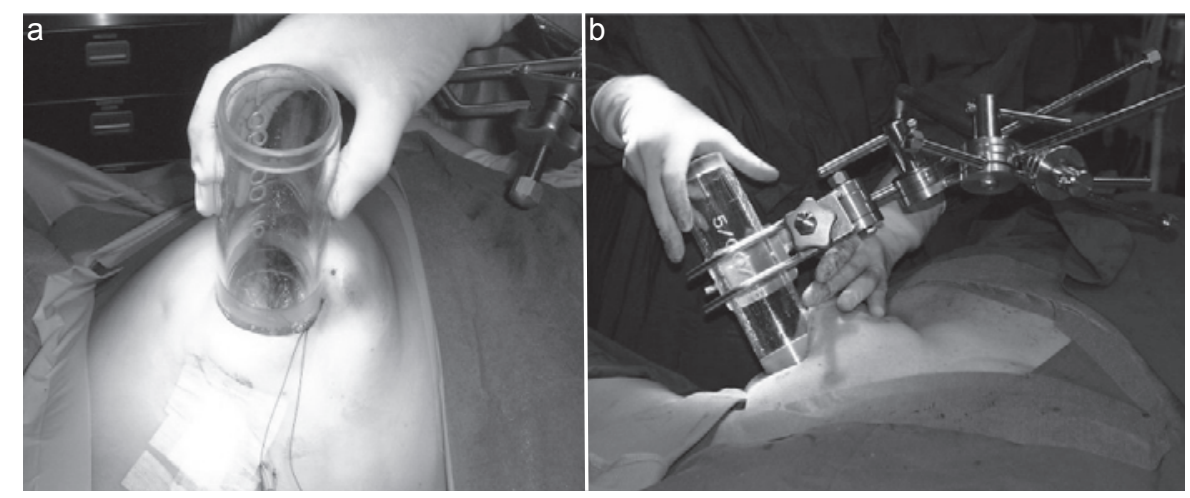

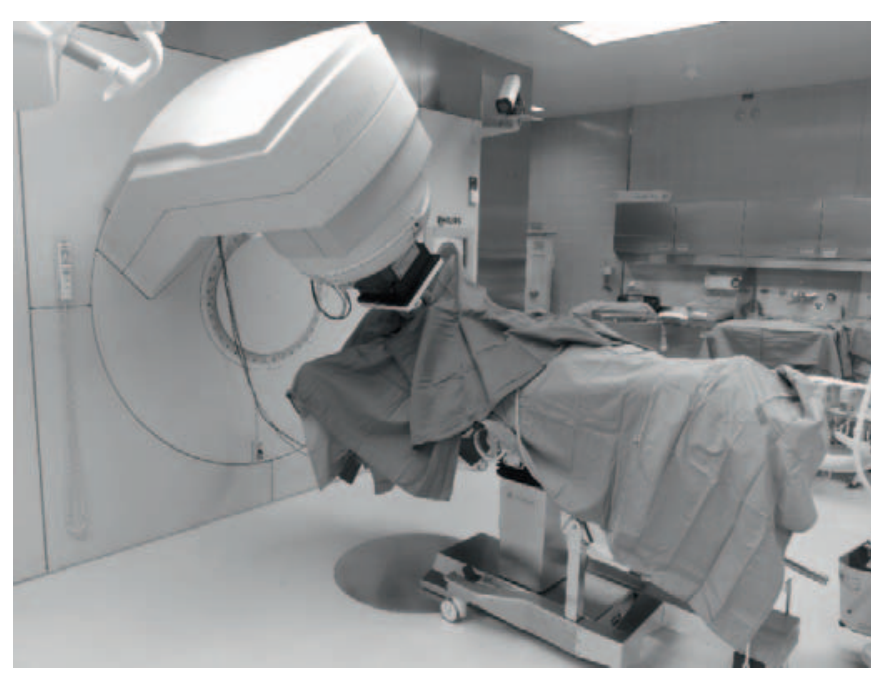

Fig. 4. Treatment position.

and $12 \mathrm{MeV}$ with a median tube size of $6 \mathrm{~cm}$. Tissue-equivalent materials like wet sterile gauzes can be used to homogenize the target surface (figs. 2-4).

\section{Clinical Results}

IOERT as a BOOST

\section{The ISIORT Pooled Analysis (BIO-Boost)}

In 2013, the ISIORT Europe (International Society of Intraoperative Radiotherapy) updated a pooled analysis of stage I-II breast cancer patients treated with an IOERT boost [17]. From 1998 to October 2005, 1,109 unselected patients of any risk group were analyzed, with highly comparable methods, sequencing, and doses for intra- and postoperative radiotherapy having been used:

A median IOERT dose of 10 Gy was applied (90\% reference isodose), using round tubes with a diameter of 5-8 $\mathrm{cm}$. WBI was prescribed with 50-54 Gy (single doses 1.7-2 Gy) and started with a median delay of 7.5 weeks post IOERT.

After a median follow-up of 72.4 months (range 0.8-239 months), only 16 in-breast recurrences were observed, half of them in the index quadrant (classified as true local recurrences), thus re- sulting in an in-breast tumor control rate of $99.2 \%$. Time to occurrence was in a range of $12.5-151$ months after primary treatment. A trend of decreasing local recurrences with rising age of the patients was seen for both in-quadrant and out-quadrant relapses. Tumor grading G3 was reported to be the only statistically relevant negative predictor for in-breast recurrence in multivariate analyses. The time between IOERT and WBI was analyzed in 3 different time slots to ascertain a possible role of a WBI delay: $<70$ days, $>70 / 140$ days, and $>140$ days. Along these time slots, no negative influence on local recurrence rates could be identified.

Furthermore, in a retrospective matched-pair analysis, 188 patients with external electron boost $(6 \times 2 \mathrm{~Gy})$ were compared to 190 IOERT patients from the Salzburg cohort [24]. At 5-year follow up, the in-breast recurrence rate in the external electron boost group was $4.3 \%$ compared to $0 \%$ in the IOERT group. This significant difference was entirely due to the reduction in true local recurrences (former index quadrant).

\section{BOOST IOERT after Primary Systemic (Neoadjuvant)}

\section{Treatment}

In several trials, higher in-breast recurrence rates are observed for patients with locally advanced breast cancer (LABC) when treated with BCS after primary systemic treatment.

In order to assess the potential of IOERT to increase local control rates also in this higher-risk setting, an institutional retrospective cohort analysis of 109 patients with LABC was performed, with local and locoregional tumor control rates as primary endpoints [19]. Patients with clinical stages II-III were treated with induction chemotherapy (mostly anthracycline/taxane-containing regimens), followed by BCS. Axillary nodes were explored by axillary dissection of levels I-II after sentinel node biopsy. 83 patients received 9 Gy IOERT during BCS (group 1); the remaining 26 patients (group 2) were treated with an external electron boost of $12 \mathrm{~Gy}$ (range 6-16 Gy) in 2-Gy fractions. All patients received adjuvant WBI of 51-57 Gy in single fractions of 1.7-1.8 Gy. The mean follow-up time in group 1 and 2 amounted to 59 months (range 3-115 months) and 67.5 months (range 13-120 months), respectively.

After a median follow-up period of 6 years, no oncological endpoint reached statistical significance, an effect mainly due to low absolute patient numbers which were not well balanced between treatment arms. However, for local control rates, there was an ab- 
solute advantage of over $10 \%$ for the IOERT group (98.5 vs. $88.1 \%$ ). Hence, these encouraging results for local control were interpreted as hypothesis-generating in that boost IOERT might be a superior strategy in these settings.

\section{Boost-IOERT in Triple-Negative Breast Cancer}

In a retrospective study [20], 71 patients diagnosed with triplenegative breast cancer (stages I-II) were investigated in terms of in-breast control and survival outcome. Radiotherapy consisted of boost IOERT with a median dose of 9.6 Gy (range 7-12 Gy) and subsequent conventionally fractionated WBI up to median total doses of $54 \mathrm{~Gy}$. Chemotherapy was applied in the neoadjuvant (12\%), adjuvant (75\%), or combinational (7\%) setting.

Triple-negativity was subclassified as 5 marker-negative (5NP) or core basal (CB) phenotype.

After a median follow-up of 97 months (range 4-170 months), survival and local control rates were evaluated. 5 in-breast recurrences in the former index quadrant were registered, whereas no regional relapses were detected. For all patients, 8-year actuarial rates for local control, metastasis-free survival, disease-specific survival, and overall-survival amounted to $89,75,80$, and $69 \%$, respectively. All local recurrences occurred in grade 3 (G3) tumors irrespective of their specific phenotype; thus, the local control rate for grade $1 / 2(\mathrm{G} 1 / \mathrm{G} 2)$ amounted to $100 \%$ for both $5 \mathrm{NP}$ and $\mathrm{CB}$, while for $\mathrm{G} 3$ it was $88 \%$ for $5 \mathrm{NP}$ and $90 \%$ for $\mathrm{CB}$ ( $\mathrm{p}=0.65$ and 0.82 , respectively; not significant). For disease-specific survival, only the difference between the best prognosis 5NP/G3 cohort versus the worst prognosis $\mathrm{CB} / \mathrm{G} 1 / 2$ cohort was statistically significant: 90 versus $54 \%(\mathrm{p}=0.03)$.

In summary, boost IOERT for triple-negative breast cancer provided good long-term local control, comparing favorably to historical control rates. In addition, IOERT seems to prolong the time interval to in-breast relapse.

\section{IOERT as $P B I$}

\section{ELIOT}

From November 2000 to December 2007, 1,305 patients between 48 and 75 years of age and with tumors smaller than $2.5 \mathrm{~cm}$ were recruited [23]. Patients were randomized to receive either single-dose IOERT with $21 \mathrm{~Gy}$ (90\% isodose) as PBI (experimental arm) or adjuvant WBI with $50 \mathrm{~Gy}$ in 25 fractions followed by an external electron boost of $10 \mathrm{~Gy}$ in 5 fractions (standard arm).

Patients with a positive sentinel node received axillary dissection. Patients with 4 or more positive axillary nodes were additionally treated with regional node irradiation up to a total dose of 50 Gy in conventional fractionation.

After a median follow-up of 5.8 years, local in-breast control was reported as primary endpoint. With regard to their topographic occurrence, local failures were registered as either relapses in the former tumor index quadrant ('real local recurrences') or 'secondary cancers' if located elsewhere in the breast. After fulldose IOERT, significantly more in-breast recurrences were noted $(\mathrm{n}=35 ; 5$-year rate: $4.4 \%)$ than after WBI $(\mathrm{n}=4 ; 5$-year rate: $0.4 \%)$ $(\mathrm{p}<0.0001)$. This was true for both in-quadrant $(2.5 \mathrm{vs.} 0.4 \%$; $\mathrm{p}=$ 0.0003 ) and out-quadrant ( 1.9 vs. $0 \% ; \mathrm{p}=0.0001)$ local failures as well as for relapses in regional nodes ( $1 \%$ vs. $0.3 \%$; $=0.03)$. However, no significant difference in overall survival was observed, and acute toxicity was lower in the experimental ELIOT arm.

In a multivariate analysis for negative predictors, the highest risk for in-breast recurrence in the ELIOT arm was seen in patients with the following characteristics: tumor size $>2 \mathrm{~cm}, 4$ or more positive lymph nodes, G3, and hormonal or triple-negative subtypes. Patients with at least 1 of these factors $(n=199)$ had a significantly higher risk of recurrence $(11.5 \%, \mathrm{p}<0.0001)$ compared to those who had none $(\mathrm{n}=452,1.5 \%)$.

Therefore, and in accordance with recent international guidelines, full-dose PBI seems to be a good treatment option for properly selected low-risk breast cancer patients classified as 'suitable' or 'good' candidates according to the ESTRO/ASTRO guidelines, with reported 5-year recurrence risks below $2 \%$ [21, 22, 25].

\section{Toxicity and Cosmetic Outcome Following Boost IORT}

So far, dose applications with intraoperative techniques have shown excellent treatment tolerance with no increased rates of acute adverse effects such as delayed wound healing or infection rates in comparison to conventional external radiotherapy [7, 26-28].

The incidence of late reactions like fibrosis/sclerosis varies slightly according to the treatment concept. Excellent results were described for boost patients with 20-25\% G1-2 and less than $2 \%$ G3 reactions [7, 29-31]. After full-dose IORT, 80\% G1, 30\% G2, and up to $6 \%$ G3 ratings have been reported [32-34].

Four reports including about 500 patients have analyzed the cosmetic outcome after boost IORT [15]. In 2 smaller trials, no difference was noted for boost IORT patients compared to conventional radiotherapy: $86 / 91 \%$ good or excellent ratings for the IORT group and $81 / 96 \%$ for the control group [27, 30].

The longest follow-up of late reactions after IOERT was published by Lemanski et al. [29] with a median follow-up time of 9 years. In all 42 recurrence-free patients, the overall cosmetic outcome was scored to be good or excellent, with 14\% G2 subcutaneous fibrosis in the former boost area.

In the Salzburg series, cosmetic long-term results were evaluated after a median follow-up of 45 months [35]. 403 patients who received boost IOERT with 10 Gy during BCS were assessed by photodocumentation in 4 standardized positions. A 5-point score described by van Limbergen [36] was used to determine the cosmetic outcome by subjective (patient) and objective (physician) rating. Furthermore, patient-, tumor- and treatment-related factors were analyzed with regard to a possible impact on the cosmetic result.

Patients' self-assessment yielded around 93\% 'satisfactory' (excellent/good) and 98\% 'acceptable' (excellent/good/moderate) results, whereas physicians scored the cosmetic outcome as satisfactory/acceptable in $64 / 95 \%$, respectively. Of note, telangiectasia was not described at all. In a separate subgroup analysis of 261 of these patients with a median follow-up of 56 months, the results were 
nearly the same: subjective assessment 91/97\% satisfactory/acceptable, and objective assessment identically with whole-group results.

Higher age and applicator diameter (possibly as surrogate for length of surgical scar) had a significant negative impact on the cosmetic outcome, whereas, for example, tumor stage, grading, electron energy, and boost volume had no significant influence.

In all these studies, different standardized cosmetic scoring systems based on qualitative estimations were used. Nonetheless, compared to conventional techniques, no negative impact on cosmetic outcome has been reported for either IOERT concept thus far.

\section{Ongoing Trials: HIOB}

Reducing the overall treatment duration without compromising local control rates is the main goal of the multicenter HIOB trial (ClinicalTrials.gov identifier: NCT01343459) which started recruitment in January 2011. Stage I/II breast cancer patients are treated with boost IOERT of $10 \mathrm{~Gy}$, followed by hypofractionated WBI $(15 \times 2.7$ Gy). Previously, the Milano Group had tested a similar phase II study design [26].

In the HIOB trial, 5-year recurrence rates are analyzed in comparison to the best published evidence in non-IORT cohorts [18]. Evidence of superiority is given if the rates fall below the nonIORT results. Secondary endpoints are disease-free survival, toxicity, and cosmetic outcome.

By October 2015, 799 patients had been recruited in 13 institutions. 695 of them are already in follow-up. At the end of WBI, $91 \%$ of the patients showed no reaction or only faint erythema, and 93\% had similar results after 4 weeks. G0-G1 late reactions (Late Effects Normal Tissue/Subjective Objective Management Analytic (LENT-SOMA) system) were recorded after 4-5 months up to 4 years after WBI in 95\%. Cosmetic outcome was assessed postoperatively by double evaluation and scored predominantly as good or excellent. After a median follow-up of 16 month (range 0.13-51 months), 4 patients had developed metastasis and 2 had died; to date, no in-breast recurrence has been noted.

In sum, the HIOB concept showed excellent results in terms of acute and late toxicity as well as cosmetic outcome, with yet no locoregional recurrence after short-term follow-up.

\section{Conclusion}

Compared to other boost methods, intraoperative treatment has clear advantages in terms of precise dose delivery, sequencing of radiation, exploiting tumor biology, and providing patient comfort.

- Direct visualization of the tumor bed during surgery guarantees accurate dose delivery. This fact has additionally gained importance in the era of primary reconstruction techniques after lumpectomy to optimize cosmetic outcome. IORT is performed before breast tissue is mobilized for plastic surgery purposes. The possibility of full skin sparing as well as the small treatment volume lead to good cosmetic and late toxicity outcomes.

- Due to the immediate irradiation after tumor removal, repopulation with possible residual cancer cells can be avoided (no 'temporal miss').

- IORT induces biological effects where the proliferative cytokine cascade in surgical wound fluid is impaired.

- IOERT prolongs the surgical procedure to a small degree; however, it shortens or, in special cases with a low risk of recurrence, even replaces adjuvant radiotherapy, creating an enormous advantage in terms of treatment comfort for the patient.

\section{Disclosure Statement}

The authors do not have any conflict of interest to declare.

\section{References}

1 Sedlmayer F, Sautter-Bihl ML, Budach W, et al: DEGRO practical guidelines: radiotherapy of breast cancer I: radiotherapy following breast conserving therapy for invasive breast cancer. Strahlenther Onkol 2013;189:825-833.

2 McGale P, Taylor C, Correa C, et al: Effect of radiotherapy after mastectomy and axillary surgery on 10year recurrence and 20-year breast cancer mortality: meta-analysis of individual patient data for 8135 women in 22 randomised trials. Lancet 2014;383: 2127-2135.

3 Darby S, McGale P, Correa C, et al: Effect of radiotherapy after breast-conserving surgery on 10-year recurrence and 15-year breast cancer death: meta-analysis of individual patient data for 10,801 women in 17 randomised trials. Lancet 2011;378:1707-1716.

4 Holland R, Veling SH, Mravunac M, et al: Histologic multifocality of Tis, T1-2 breast carcinomas. Implications for clinical trials of breast-conserving surgery. Cancer 1985;56:979-990.
5 Faverly DR, Hendriks JH, Holland R: Breast carcinomas of limited extent: frequency, radiologic-pathologic characteristics, and surgical margin requirements. Cancer 2001;91:647-659.

6 Bartelink H, Maingon P, Poortmans P, et al: Wholebreast irradiation with or without a boost for patients treated with breast-conserving surgery for early breast cancer: 20-year follow-up of a randomised phase 3 trial. Lancet Oncol 2015;16:47-56.

7 Merrick HW 3rd, Battle JA, Padgett BJ, et al: IORT for early breast cancer: a report on long-term results. Front Radiat Ther Oncol 1997;31:126-130.

8 Dubois JB, Hay M, Gely S, et al: IORT in breast carcinomas. Front Radiat Ther Oncol 1997;31:131-137.

-9 Fowler JF: The linear-quadratic formula and progress in fractionated radiotherapy. Br J Radiol 1989;62:679-694.

10 Bentzen SM, Agrawal RK, Aird EG, et al: The UK Standardisation of Breast Radiotherapy (START) Trial $\mathrm{B}$ of radiotherapy hypofractionation for treatment of early breast cancer: a randomised trial. Lancet 2008; 371:1098-1107.
11 Whelan TJ, Kim DH, Sussman J: Clinical experience using hypofractionated radiation schedules in breast cancer. Semin Radiat Oncol 2008;18:257-264.

12 Bartelink H: Commentary on the paper 'A preliminary report of intraoperative radiotherapy (IORT) in limited-stage breast cancers that are conservatively treated'. A critical review of an innovative approach. Eur J Cancer 2001;37:2143-2146.

13 Belletti B, Vaidya JS, D'Andrea S, et al: Targeted intraoperative radiotherapy impairs the stimulation of breast cancer cell proliferation and invasion caused by surgical wounding. Clin Cancer Res 2008;14:1325-1332.

14 Veldwijk MR, Neumaier C, Gerhardt A, et al: Comparison of the proliferative and clonogenic growth capacity of wound fluid from breast cancer patients treated with and without intraoperative radiotherapy. Transl Cancer Res 2015;4:173-177.

15 Sedlmayer F, Reitsamer R, Wenz F, et al: Intraoperative radiotherapy (IORT) as boost in breast cancer. Radiat Oncol 2017;12:23. 
16 Herskind C, Ma L, Liu Q, et al: Biology of high single doses of IORT: RBE, 5 R's, and other biological aspects. Radiat Oncol 2017;12:24.

17 Fastner G, Sedlmayer F, Merz F, et al: IORT with electrons as boost strategy during breast conserving therapy in limited stage breast cancer: long term results of an ISIORT pooled analysis. Radiother Oncol 2013;108 279-286.

18 Fastner G, Reitsamer R, Fussl C, et al: Hypofraktionierte Ganzbrustbestrahlung mit intraoperativem Elektronen-Boost beim brusterhaltend operierten Mammakarzinom (HIOB): Aktualisierte Resultate einer prospektiv multizentrischen Studie. Strahlenther Onkol 2016;192:32.

19 Fastner G, Reitsamer R, Ziegler I, et al: IOERT as anticipated tumor bed boost during breast-conserving surgery after neoadjuvant chemotherapy in locally advanced breast cancer - results of a case series after 5-year follow-up. Int J Cancer 2015;136:1193-1201.

20 Fastner G, Hauser-Kronberger C, Moder A, et al: Survival and local control rates of triple-negative breast cancer patients treated with boost-IOERT during breast-conserving surgery. Strahlenther Onkol 2016; 192:1-7.

21 Correa C, Harris EE, Leonardi MC, et al: Accelerated partial breast irradiation: executive summary for the update of an ASTRO evidence-based consensus statement. Pract Radiat Oncol 2017;7:73-79.

22 Leonardi MC, Maisonneuve P, Mastropasqua MG, et al: Accelerated partial breast irradiation with intraoperative electrons: using GEC-ESTRO recommendations as guidance for patient selection. Radiother Oncol 2013;106:21-27.
Veronesi U, Orecchia R, Maisonneuve P, et al: Intraoperative radiotherapy versus external radiotherapy for early breast cancer (ELIOT): a randomised controlled equivalence trial. Lancet Oncol 2013;14:1269-1277.

4 Reitsamer R, Sedlmayer F, Kopp M, et al: The Salzburg concept of intraoperative radiotherapy for breast cancer: results and considerations. Int J Cancer 2006;118: 2882-2887.

25 Leonardi MC, Maisonneuve P, Mastropasqua MG, et al: How do the ASTRO consensus statement guidelines for the application of accelerated partial breast irradiation fit intraoperative radiotherapy? A retrospective analysis of patients treated at the European Institute of Oncology. Int J Radiat Oncol Biol Phys 2012;83:806813.

26 Vaidya JS, Baum M, Tobias JS, et al: Targeted intraoperative radiotherapy (Targit): an innovative method of treatment for early breast cancer. Ann Oncol 2001; 12:1075-1080.

27 Ciabattoni A, Fortuna G, Ciccone V, et al: IORT in breast cancer as boost: preliminary results of a pilot randomized study on use of IORT for Stage I and II breast cancer. Radiother Oncol 2004;73:35-36.

28 Reitsamer R, Peintinger F, Sedlmayer F, et al: Intraoperative radiotherapy given as a boost after breast-conserving surgery in breast cancer patients. Eur J Cancer 2002;38:1607-1610.

29 Lemanski C, Azria D, Thezenas S, et al: Intraoperative radiotherapy given as a boost for early breast cancer: long-term clinical and cosmetic results. Int J Radiat Oncol Biol Phys 2006;64:1410-1415.
30 Kraus-Tiefenbacher U, Bauer L, Kehrer T, et al: Intraoperative radiotherapy (IORT) as a boost in patients with early-stage breast cancer - acute toxicity. Onkologie 2006;29:77-82.

1 Ivaldi GB, Leonardi MC, Orecchia R, et al: Preliminary results of electron intraoperative therapy boost and hypofractionated external beam radiotherapy after breast-conserving surgery in premenopausal women. Int J Radiat Oncol Biol Phys 2008;72:485-493.

32 Mussari S, Sabino Della Sala W, Busana L, et al: Fulldose intraoperative radiotherapy with electrons in breast cancer. First report on late toxicity and cosmetic results from a single-institution experience. Strahlenther Onkol 2006;182:589-595.

33 Ollila DW, Klauber-DeMore N, Tesche LJ, et al: Feasibility of breast preserving therapy with single fraction in situ radiotherapy delivered intraoperatively. Ann Surg Oncol 2007;14:660-669.

34 Lemanski C, Azria D, Gourgou-Bourgade S, et al: Electrons for intraoperative radiotherapy in selected breast-cancer patients: late results of the Montpellier phase II trial. Radiat Oncol 2013;8:191.

35 Fussl C, Merz F, Fussl A, et al: Evaluation der kosmetischen Langzeitergebnisse bei Brustkrebs im Frühstadium nach intraoperativer Radiotherapie (IORT) im Rahmen der brusterhaltenden Therapie. Strahlenther Onkol 2012;188:189.

36 Van Limbergen E, van der Schueren E, Van Tongelen $\mathrm{K}$ : Cosmetic evaluation of breast conserving treatment for mammary cancer. 1. Proposal of a quantitative scoring system. Radiother Oncol 1989;16:159-167. 